\title{
Preface
}

\section{PET-CT-MR Imaging-Based Cardiovascular Imaging}

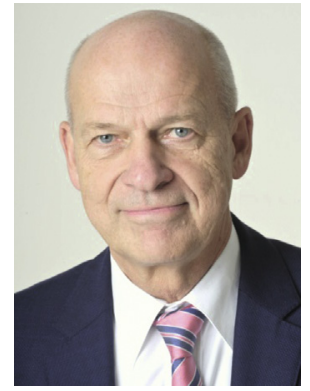

Poul Flemming HøilundCarlsen, MD, DMSc, Prof (Hon)

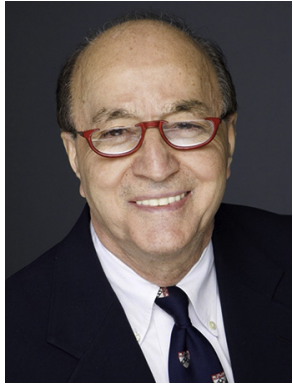

Abass Alavi, MD, MD (Hon), PhD (Hon), DSC (Hon)

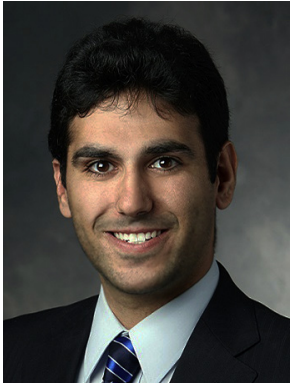

Mateen C. Moghbel, MD

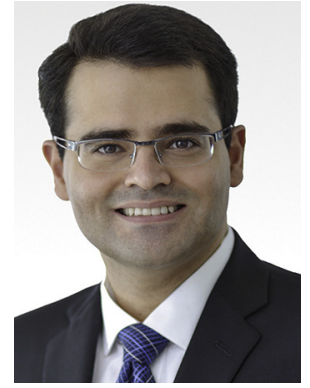

Ali Salavati, MD, MPH

Editors

The past five decades have witnessed a major revolution in medical imaging, and this has had a profound impact on the management of many maladies, including cardiovascular disorders. Conventional planar $\mathrm{x}$-ray imaging has been replaced by $\mathrm{x}$-ray-based computed tomography (CT) for many medical indications. The introduction of MR imaging has further enhanced the role of structural imaging, particularly in the assessment of diseases and disorders that have affected the central nervous, musculoskeletal, and cardiovascular systems. In parallel with the advances that have been made in the detection of structural abnormalities visualized by CT and MR imaging, the evolution of functional and molecular imaging with PET has reshaped the role of medical imaging in many domains.

As the medical community started to utilize these powerful imaging techniques on a wide scale, it became clear that the sensitivity and specificity of structural imaging modalities are often suboptimal for the early detection of disease and the assessment of response to therapy. It is typically late in a disease process when changes on a physiologic level, such as blood flow and organ motility, lead to structural abnormalities that become apparent on modalities such as CT and MR imaging (Fig. 1). Therefore, relying on structural imaging for the management of many diseases can lead to delayed and often prolonged interventions and suboptimal outcomes. By contrast, molecular probes, which visualize pathology on a microscopic rather than macroscopic level, have the potential to detect disease and allow for intervention earlier in the process.

Cardiovascular imaging with radiotracer-based approaches has been primarily based on compounds that are labeled with single gamma emitters. While early applications of perfusion imaging techniques utilized planar data acquisition, over the past three decades such studies have been carried out by employing singlephoton emission computed tomography (SPECT). Currently, SPECT perfusion imaging is the most commonly used imaging modality in the field of nuclear medicine for assessing atherosclerosis in the coronary arteries. However, SPECT imaging instruments suffer from poor spatial resolution and suboptimal quantification. Therefore, the sensitivity and specificity of this approach are somewhat limited, and as such, there is a dire need to introduce newer techniques with better performance.

During the past four decades, PET imaging with novel radiotracers has revolutionized the specialty of medical imaging. While initial applications of PET were confined to evaluating central nervous system disorders, with the introduction of advanced PET machines, it has become feasible to image every organ system in the body. Particularly, PET has become the imaging modality of choice in the management of a large number of 


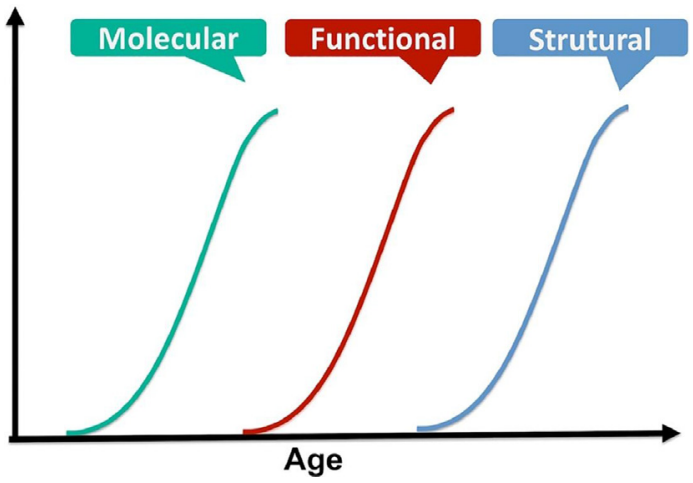

Fig. 1. The probable sequence of biological events as they relate to many disorders. Functional activities refer to physiological alterations, such as blood flow and organ motility. This pattern is particularly relevant to the assessment of atherosclerosis in the coronary arteries as well as other arteries. As such, molecular imaging with PET may provide early evidence of the disease process. (From Moghbel M, AlZaghal A, Werner TJ, et al. The role of PET in evaluating atherosclerosis: a critical review. Semin Nucl Med 2018;48(6):489; with permission.)

malignancies. To date, the workhorse for PETbased assessment of cancer has been ${ }^{18} \mathrm{~F}$-fluorodeoxyglucose (FDG). However, widespread use of this technology has revealed its nonspecificity for cancer due to high uptake of FDG in inflammatory lesions. Attempts have been made to examine a variety of inflammatory disorders, including atherosclerotic plaques in the major arteries. However, the nonspecificity of FDG for detecting inflammation in the plaques has posed a significant challenge for visualizing and characterizing atherosclerosis in the arteries. Moreover, intense uptake of FDG in the myocardium has made it impossible to use this technique to detect inflammatory plaques in the coronary arteries. Therefore, other tracers have been introduced for assessing a variety of cardiovascular disorders, with an emphasis on atherosclerosis. Among these tracers, ${ }^{18} \mathrm{~F}$-labeled sodium fluoride (NaF) appears to be of particular promise for detecting molecular calcification in this degenerative process. This tracer is specific and also sensitive for the detection of molecular calcification in plaques at various stages of the disease. The preliminary data from many centers appear to be very promising, and as such, this approach may substantially improve our ability to assess patients with suspected or proven atherosclerosis and determine the impact of various interventions (Fig. 2). The role of other tracers that have been tested over the years is somewhat uncertain at this time due to the suboptimal spatial resolution of PET instruments in detecting subtle pathologic changes in atherosclerotic lesions.

The introduction of PET-CT in 2001 has further enhanced the role of molecular imaging in the management of patients with cardiovascular disorders. X-ray-based CT has been extensively employed to detect structural calcification in the coronary and major arteries over the years. It is our view that structural calcification as noted on $\mathrm{CT}$ is of limited value since it reveals irreversible stages of the disease. When such abnormalities are visualized on CT scan, it is very likely that the disease process has been ongoing for decades and has therefore led to permanent damage. The data generated by adopting NaF-PET imaging suggest that this technique may become invaluable in the management of patients with coronary and other major artery atherosclerosis. In the past two decades, multislice CT has been employed to perform coronary arteriography with success. This methodology allows detection of vascular narrowing due to advanced atherosclerotic plaques. This has substantially eliminated the need for catheterbased arteriography, which was considered the gold standard prior to the introduction of cardiac CT. We believe the combination of PET with CT will substantially enhance the performance of both techniques in the detection and quantification of atherosclerotic lesions.

The introduction of MR imaging as a major imaging modality in the 1980s has further enhanced the role of imaging for examining cardiovascular disorders. MR imaging has been of great value in assessing soft tissue abnormalities in the cardiovascular system as well as in other organs in the

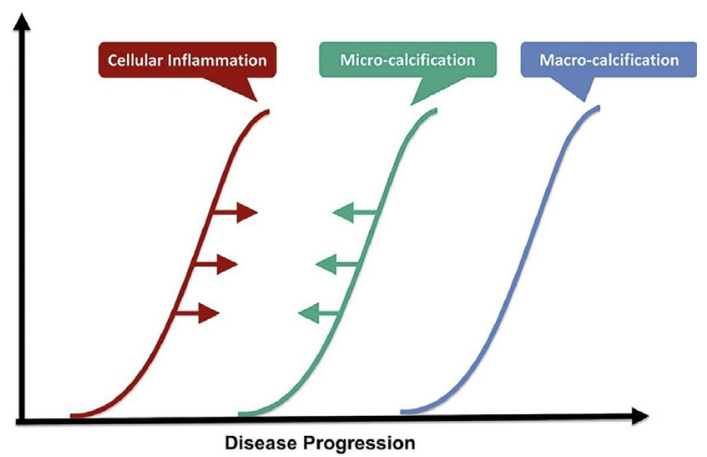

Fig. 2. The probable sequence of biological events in atherosclerosis. It is well known that endothelial inflammation and vascular microcalcification precede macrocalcification; thus, FDG and NaF PET could detect the earliest phases of the disease even in asymptomatic individuals. (From Moghbel M, AlZaghal A, Werner TJ, et al. The role of PET in evaluating atherosclerosis: a critical review. Seminin Nucl Med 2018;48(6):489; with permission.) 
body. This is in contrast with CT imaging, which has been of limited value in this domain. Therefore, MR imaging for detecting cardiac and vascular abnormalities has played a major role in managing disorders that occur in the cardiovascular system. Claims have been made about the role of MR imaging in assessing disease processes on the molecular and cellular levels. Unfortunately, the limited sensitivity of this modality has impeded its success, and therefore, its impact as a molecular imaging probe with current instruments. However, PET-MR imaging as a combined modality will substantially enhance the role of each modality by visualizing and further characterizing abnormalities that are detected by PET.

Finally, the limited field of view of the current PET instruments has been an obstacle for optimal utilization of this technique in both clinical and research domains. In other words, the limited axial field of view requires 20 to 25 minutes of imaging within 1 to 2 hours following the administration of compounds such as FDG. High background activity in the vascular system during the first 2 hours adversely affects the sensitivity of this technique in detecting atherosclerotic plaques. Therefore, instruments that allow for imaging of the entire body simultaneously may further enhance the role of PET in examining cardiovascular disorders. In the past few years, attempts have been made to image the entire body with a substantially larger field of view and greatly enhanced sensitivity. The success of these efforts will strengthen the impact of PET imaging in this extremely prevalent disease. This approach allows for total body imaging in 3 to $5 \mathrm{mi}-$ nutes, 3 to 4 hours after the administration of molecular compounds, when the background activity has substantially decreased and is less of a barrier to accurate quantification of atherosclerotic burden.
In this issue of PET Clinics, we have included scientific communications that are currently of great interest to the medical community with regard to the performance of PET imaging techniques for both clinical and research applications.

Poul Flemming Høilund-Carlsen, MD, DMSc, Prof (Hon)

Department of Nuclear Medicine Odense University Hospital and Department of Clinical Research University of Southern Denmark Sønder Boulevard 29 Odense, 5000 Denmark

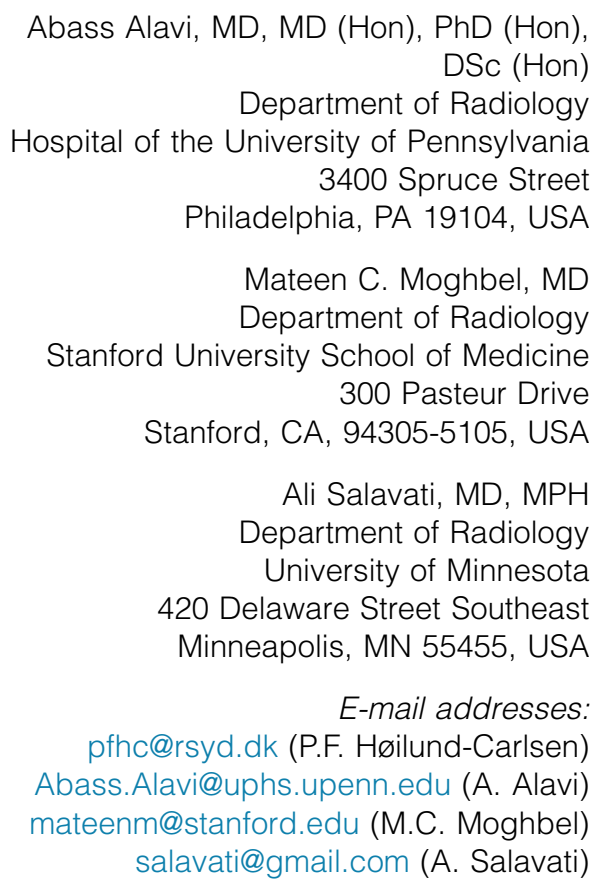

E-mail addresses:

pfhc@rsyd.dk (P.F. Høilund-Carlsen) Abass.Alavi@uphs.upenn.edu (A. Alavi) mateenm@stanford.edu (M.C. Moghbel) salavati@gmail.com (A. Salavati) 